\title{
THEORETICAL ANALYSIS OF A HYDRAULIC DRIVE SYSTEM. THE INFLUENCE OF THE WORK ENVIRONMENT ON THE PERFORMANCE OF THE SYSTEM
}

\author{
Mihai Avram ${ }^{1}$, Mariana-Florentina Ştefănescu ${ }^{1}$, Valerian-Emanuel Sârbu ${ }^{1}$, Gabriel Năstase ${ }^{2}$ \\ ${ }^{1}$ University Politehnica of Bucharest, Faculty of Mechanical Engineering and Mechatronics, \\ 313 Splaiul Independentei, 060042, Bucharest, Romania \\ ${ }^{2}$ Transilvania University of Brasov, 29 Bd-ul Eroilor, Brașov, România \\ E-mails: mavram02@yahoo.com, mariana.stefanescu@upb.ro, vale sarbu@yahoo.com, traznasa@gmail.com
}

\begin{abstract}
This paper aims at highlighting the influence of the properties of the working medium used on static and dynamic performance of intelligent hydraulic drive systems. This paper briefly presents the laws and equations commonly used in the theoretical analysis of pressure fluid systems. Basically, we have aimed at providing a review of these equations and concepts required to develop the mathematical model of such a system.
\end{abstract}

Keywords: Hydraulic Oil, Density, Hydraulic Drive System, The Theoretical Analysis.

\section{Introduction}

Hydraulic drive systems are used in large scale industrial, mobile and aircraft hydraulic applications due to some advantages, of which we mention: they can be extremely energy efficient, easy to maintain and silent [1].

Static and dynamic quality performance of a hydraulic drive system can be determined theoretically or experimentally.

The theoretical analysis assumes achievement of a "model" for the system as a first step. The model of the analyzed system is another system, equivalent in some ways to the first, but easier to study; the information provided by the study of the model provides accurate definition of the system behavior [2], [3].

The study of real fluid flow is generally difficult to address by complete theoretical methods, due to the special complications caused by a number of disturbing factors such as fluid friction (viscosity), turbulence, driving solid particles, presence of internal heat sources etc. The knowledge of fundamental properties behavior in a system can help us understand the nature of molecular interactions and so we can determine other thermodynamic properties [4], [5], [16], [17]. Temperature and density are factors that affect the treated metals, which have an important influence on the macroscopic mechanical properties and the stability of materials [6], [7]. For this reason, nonessential disturbing factors are frequently eliminated, and are kept only those whose effect is decisive.
By doing so, we reached the development of simplified theoretical models that allow accurate description of most of processes related to the dynamics of real fluid flow.

In the case of hydraulic systems, the working medium is the fluid used as energy and informationcarrying agent. As a work medium, we used mineral or synthetic oil that may contain some additives used to improve the characteristics or to adapt the oil to the chosen application.

In the case of drive systems operating with a fluid working medium, we consider a model of the working medium. Model of the working medium means an idealized fluid which is attributed to the main macroscopic properties of the real fluid, neglecting some secondary aspects of the flow phenomena.

The most important models currently used [8] are:

a) the ideal non-viscous fluid (Euler), with a negligible viscosity and that does not adhere to solid surfaces it is in contact with;

b) the ideal viscous fluid (Newton), that adheres to solid surfaces it is in contact and to which the viscous tension (fluid friction) depends on the rate of deformation of the medium through a linear type relation;

c) the homogeneous fluid, which has the same density at each time $t \rho$ in all the points of the field occupied by it;

d) the incompressible fluid, whose density $\rho$ is independent of time $t$ at all points of the domain occupied by it; 
e) the compressible fluid, whose density $\rho$ depends on the time $t$ and / or the position of the explored point within the domain occupied by it;

f) the light fluid, whose behavior is not significantly influenced by the action of the gravitational force field;

g) the heavy fluid, whose behavior is significantly influenced by the action of the gravitational force field.

Based on our model, we can proceed to the elaboration of the mathematical model of the analyzed system [9]. This is actually an idealized representation of the physical system consisting of mathematical or logical functions that describe the status of system variables and of how variables change and interact with each other.

For simple systems, the mathematical models obtained can be analytically analyzed. In the case of complex systems, mathematical models that are impossible to solve through conventional analytical methods are obtained. In the past, these issues have only been analyzed theoretically; mathematically it was possible to prove that their solution exists and is unique but finding it by classical methods was impossible. We have used approximations that led to mathematical models that no longer rigorously represented the studied system; the resulting solution is characterized by a high degree of imprecision. Emergence of computers ensured fast, accurate and low-cost solutions to issues that were considered unapproachable. In fact, mathematical simulation and computer-based simulation became working techniques indispensable in modern research and design activities in the most diverse fields.

\section{Basic Equations}

The mathematical models of systems working with fluid medium contain a series of equations where the fluid intervenes through a set of parameters such as: fluid density $(\rho)$, fluid modulus of elasticity (E), dynamic or kinematic viscosity $(\eta-\mu)$. Below, the equations in which these parameters occur are identified.

The flow equation, is the equation that actually expresses the law of mass preservation [1]. According to this law, the difference between the mass of fluid entering $\left(\dot{m}_{i}\right)$ and the mass of fluid coming out $\left(\dot{m}_{e}\right)$ of a volume (Fig. 1) in the unit of time is equal to the variation of the mass of the fluid $\mathrm{M}$ contained in the volume $\mathrm{V}$ (volume located inside an equipment or a connecting pipe).

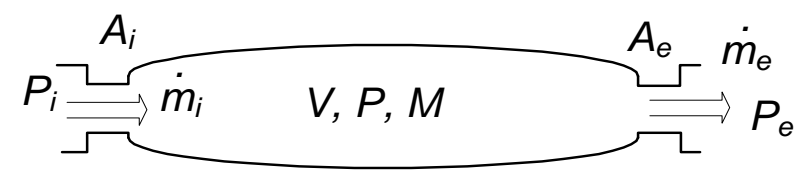

Figure 1: Hydraulic circuit - detail

The equation for mass preservation in the most general form can be written:

$$
\dot{m}_{i}-\dot{m}_{e}=\frac{d M}{d t}
$$

By derivation of $\mathrm{M}$ and the replacement in the above relation of the mass flows with the volumetric flows is obtained the relation:

$$
\begin{array}{ll}
q_{i}-q_{e}=\frac{d V}{d t}+\frac{V}{\rho} \cdot \frac{d \rho}{d t} & \text { or } \\
\frac{d P}{d t}=\frac{E}{V} \cdot\left(q_{i}-q_{e}\right)-\frac{E}{V} \cdot \frac{d V}{d t} &
\end{array}
$$

the volumetric flows, in the conditions where the flow through the input and output sections in volume $\mathrm{V}$ is turbulent, have the expressions:

$$
\begin{aligned}
& q_{i}=\alpha \cdot A_{i} \cdot \sqrt{\frac{2}{\rho} \cdot\left(P_{i}-P\right)} \\
& q_{e}=\alpha \cdot A_{e} \cdot \sqrt{\frac{2}{\rho} \cdot\left(P-P_{e}\right)}
\end{aligned}
$$

where $\alpha$ is the flow rate [1].

\section{Equations of motion}

Most of the equipment that is part of the hydraulic drive systems has structural elements or sub-assemblies in their structure moving or in balance under the effect of forces or moments acting on them. Exception is the fluid equipment without moving parts.

The equation of motion expresses the balance of forces or moments acting on movable elements in hydraulic automation equipment.

By their nature these components can be divided into: mechanical, electrical or electromagnetic and due to the working fluid medium.

In terms of issues considered of interest are the forces of the last category, of which the flowing ones are the most suggestive. These forces occur in the majority of hydraulics as a consequence of local change in the flowing sections (and thus of acceleration or deceleration of fluid masses) or change in the flow direction. In both situations the flow rate changes the module and / or the direction, which results into a variation of the movement amount. 
For example, Fig. 2 considers to be a feeder with a cylindrical drawer with translation movement [1], [10]; through its axial position the dispenser drawer

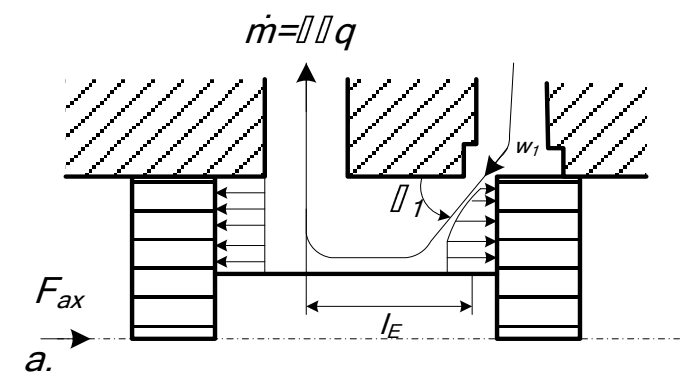

controls the fluid flow $q$ entering into the feeder(Fig.2.a), and coming out of the feeder (Fig. 2.b).

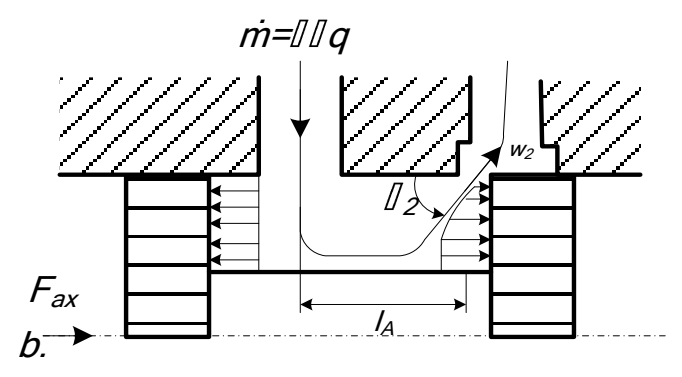

Figure 2: Hydraulic distributor with cylindrical drawer with translational motion; (a) the input section is the control and (b) the output section is the control

For these two situations we can write the expression of force flow as follows:

$$
\begin{aligned}
& F_{c x}=\rho \cdot q \cdot w_{1} \cdot \cos \theta_{1}-\rho \cdot l_{E} \cdot \frac{d q}{d t} \quad \text { and } \\
& F_{c x}=\rho \cdot q \cdot w_{2} \cdot \cos \theta_{2}-\rho \cdot l_{A} \cdot \frac{d q}{d t}
\end{aligned}
$$

\section{Friction forces in seals}

In the construction of equipment components of the hydraulic drive systems are sealing elements that may have one of the following functions: air-tight isolation of volumes containing fluid under pressure, separation of the active cameras of the engines, normally loaded with variable frequencies, removal of fluid losses in certain volumes and preventing ingress of external impurities in the volume.

We may speak of a "live" seal - metal on metal or a sealing with special seals. Of interest are movable seals made between a fixed part and a movable one). Often these seals are made at the level of some cylindrical parts (due to the ease of machining thereof).

The viscous friction force $F_{\eta}$ (Fig. 3) acting on the movable item is determined by the relationship:

$$
F_{\eta}=\pi \cdot d_{p} \cdot l \cdot \eta \cdot \frac{d v}{d y}
$$

Where $d v / d t$ is the speed gradient and, $\eta$ the dynamic viscosity of the fluid.

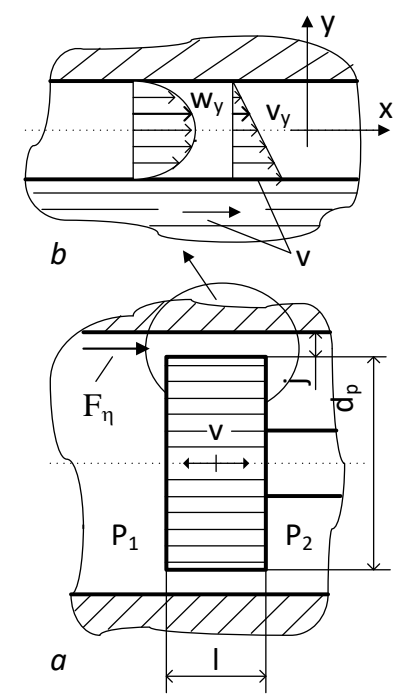

Figure 3: (a) The viscous friction force when moving a cylindrical mobile element assembly view (b) detail

The viscous friction force at the piston level [11] is obtained for $\mathrm{y}=-\mathrm{j} / 2$ and has the expression:

$F_{\eta}=\pi \cdot d_{p} \cdot\left(\frac{\Delta p}{2} j \mp \frac{l \cdot \eta}{j} \cdot v\right)$

\section{Oil density}

In the case of oils, density is defined as follows:

$$
\rho=\frac{\text { Mass }}{\text { Volume }}=\lim _{\Delta V \rightarrow 0} \frac{\Delta m}{\Delta V}=\frac{d m}{d V}
$$

The density of hydraulic fluids is normally between $850 \ldots 910 \mathrm{Kg} / \mathrm{m}^{3}$. 
Since the volume of a fluid changes with the temperature and the pressure it appears that density is a function of the same parameters as follows:

- at constant pressure, when the temperature of a fluid increases, the expansion phenomenon occurs, the fluid will occupy a larger volume and consequently its density decreases;

- at a constant temperature, if the pressure increases, a compression phenomenon occurs, the fluid will occupy a smaller volume and consequently increases its density.

This function is based on state equation [12], which links the measures: pressure $p$, density $\rho$ and temperature t. For many substances the state equation is too complex to be easily used or contains coefficients that have not been accurately measured. In the case of fluids, the state equation has a more complex form, but can be linearized.

In a first approximation, fluids are considered incompressible, thus for normal calculations the fluid density is considered constant within the usual temperature and pressure limits.

To get good precision, density should be expressed as a function of temperature and pressure. The actual physical state of the fluid at an arbitrarily chosen point in the fluid mass is considered to be expressed by a form equation:

$$
\rho=\rho(p, t)
$$

where $\mathrm{p}, \rho$ and $\mathrm{t}$ is the pressure, density, or absolute local temperature. The relationship (8) is referred to as the state of the fluid considered.

The function (8) developed in Taylor series around the operating point $\left(\mathrm{p}_{0}, \mathrm{t}_{0}\right)$ and approximated by the first three terms leads to:

$$
\rho \approx \rho_{0}+\left(\frac{\delta \rho}{\delta p}\right)_{t}\left(p-p_{0}\right)+\left(\frac{\delta \rho}{\delta t}\right)_{p}\left(t-t_{0}\right)
$$

Two situations are usually considered:

1. The process is isothermal, that is, if the temperature remains constant $\left(t=t_{0}\right)$; in this case the relation (9) becomes:

$$
\rho=\rho_{0} \cdot(1+\beta \cdot \Delta p)=\rho_{0} \cdot\left(1+\frac{1}{E} \cdot \Delta p\right)
$$

where $\beta=\frac{1}{\rho_{0}}\left(\frac{\delta \rho}{\delta p}\right)_{t}\left[\mathrm{~m}^{2} / N\right]$ is the isothermal compressibility coefficient, E - "isothermal bulk moduls" or "bulk moduls", and $\Delta p=p-p_{0}$.

For mineral oils considering the temperature varies in the range $-40 \ldots 120^{\circ} \mathrm{C}$, and the pressure does not exceed 320 bar we can adopt an average value for E within the range $14000 \ldots 16000$ bar. However, this approximation is not satisfactory, which is why, in literature, the relationship is proposed:

$$
E=E+k_{p} \cdot p .
$$

Frequently is adopted: $\mathrm{E}=16000 \mathrm{bar}, \mathrm{k}_{\mathrm{p}}=9,558$. Influence of temperature on the elasticity is negligible.

2. The thermodynamic process is isobar, that is, the pressure remains constant $\mathrm{p}=\mathrm{p}_{0}$; in this case the relationship (9) becomes:

$$
\rho=\rho_{0} \cdot(1-\alpha \cdot \Delta t)
$$

where $\alpha=-\frac{1}{\rho_{0}}\left(\frac{\partial p}{\partial T}\right)_{P=P_{0}}$ is the coefficient of isobaric expansion, and $\Delta \mathrm{t}=\mathrm{t}-\mathrm{t}_{0}$.

Density variation with the pressure and temperature can be expressed based on relations (10) and (12) following a law of form:

$$
\rho=\rho_{0} \cdot(1-\alpha \cdot \Delta t+\beta \cdot \Delta p)
$$

The literature [1], [12]-[14] provides several relationships that establish dependence of density with temperature and pressure in the case of mineral oils. Here are some examples in this regard:

$$
\begin{aligned}
& \rho_{t}=\frac{\rho_{0}}{1+0,00064 \cdot(t-15)} \quad \text { or } \\
& \rho_{t}=\rho_{0} \cdot[1-0,00064 \cdot(t-15)]
\end{aligned}
$$

Where $\rho_{0}$ is the density of the oil at temperature $\mathrm{t}=15^{\circ} \mathrm{C}$; the relationship is valid if the pressure is atmospheric, ie $\mathrm{P}=1,013 \cdot 10^{5} \mathrm{~N} / \mathrm{m}^{2}$;

$\rho_{p}=\rho_{0} \cdot\left[1+0,000075 \cdot\left(p-p_{0}\right)\right]$

For a better understanding of the issue in question let $\mathrm{s}$ consider the concrete case of high viscosity hydraulic oil, category ISO-L-HV - grade ISO 100 , oil that can be used in high-performance hydraulic drive systems. Some of the features of this type of oil, for different ISO grades, according to the technical data provided by the manufacturer, are presented in Table 1.

Table 1.

Oil "ISO-L-HV-ISO-100" hydraulic characteristics

\begin{tabular}{|l|l|l|l|l|l|l|l|l|}
\hline Characteristic & Unit & Method & \multicolumn{4}{|l|}{ Average values } & \multicolumn{3}{l|}{$\mid$} \\
\hline ISO grades & - & - & 15 & 22 & 32 & 46 & 68 & 100 \\
\hline Volume mass at $15^{\circ} \mathrm{C}$ & $\mathrm{kg} / \mathrm{m}^{3}$ & NF T $60-101$ & 860 & 865 & 870 & 870 & 875 & 877 \\
\hline Kinematic viscosity at $40^{\circ} \mathrm{C}$ & $\mathrm{mm}^{2} / \mathrm{s}$ & NF T $60-100$ & 14.9 & 23.7 & 35.0 & 48.4 & 69.0 & 107.4 \\
\hline Kinematic viscosity at $100^{\circ} \mathrm{C}$ & $\mathrm{mm}^{2} / \mathrm{s}$ & NF T $60-100$ & 3.8 & 5.1 & 6.7 & 8.4 & 11 & 15.1 \\
\hline
\end{tabular}


For the oil considered, we shall have $\rho_{0}=877$ $\mathrm{kg} / \mathrm{m}^{3}$. Instead, depending on the system load, the pressure may vary within wide limits. Under these conditions, according to the relationship (15) the density will be given by the relationship:

$$
\rho_{p}=\rho_{0} \cdot\left[0.968+0.000064 \cdot\left(P-P_{0}\right)\right]
$$

Figure 4 graphically shows the functions $\rho_{\mathrm{t}}(\mathrm{t})$ and $\rho_{\mathrm{p}}(\mathrm{P})$ defined by relationships (14), (15) and (16).

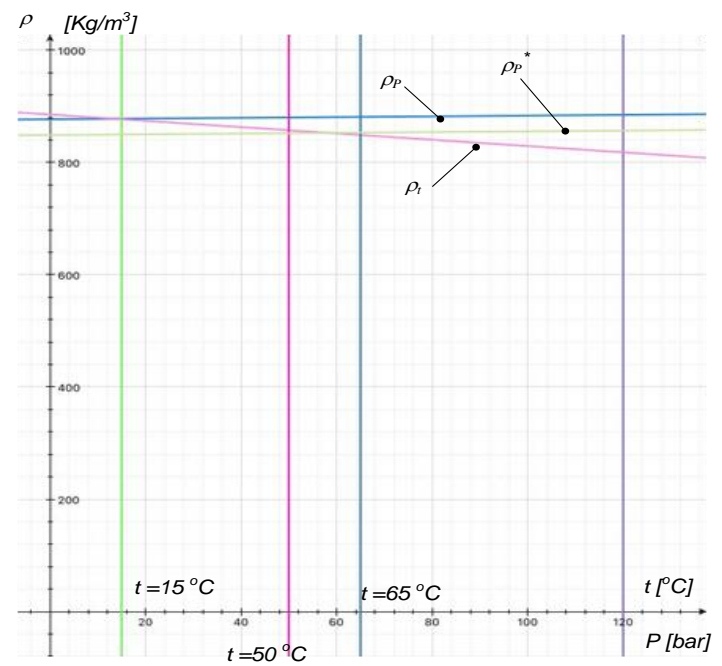

Figure 4: Density variation with temperature and pressure for a certain type of oil

\section{Oil viscosity}

Viscosity is the property of real fluids to resist relative displacement of their particles. It therefore manifests itself only during the movement of fluids through emergence of internal friction forces, tangent to the fluid layers, also called viscous forces.
According to Newton's law, the tangential forces that take between two adjacent fluid layers $d z$ which travel at a relative speed $d w_{x}$ are proportional to the gradient of the speed of the layers and the contact surface:

$$
d F=\eta \cdot d A \cdot \frac{d w_{z}}{d z}
$$

Where: $d A$ represents the elementary surface of the contact layer, $d w_{x} / d z$ speed gradient, and $\eta$ the coefficient of proportionality, called also dynamic viscosity. In some applications the kinematic viscosity coefficient $v=\eta / \rho$.

Oil viscosity depends on its temperature and pressure. This dependence is important not only in the case of a theoretical analysis, but in terms of practical behavior of the actual hydraulic systems where the two parameters, temperature and pressure, change continuously.

Hydraulic oil viscosity is extremely sensitive to operating temperature. At a growing temperature, oil viscosity drops rapidly. Oil viscosity at a specified temperature (Table 2) can be calculated using a series of empirical or derivative theoretical models. One example in this regard [15] is the relation:

$$
\eta=\eta_{0} \cdot e^{-\lambda_{1} \cdot\left(t-t_{0}\right)}
$$

Where $\eta_{0}$ is the dynamic viscosity at temperature $t_{0}$, and $\lambda_{1}$ is a coefficient determined experimentally for each oil used. For mineral oils, it is in the range [0.036, 0.057] $\mathrm{K}^{-1}$.

Table 2 also shows other equations found in the literature [14] under different names.

Table 2. Dynamic viscosity with temperature correlation

\begin{tabular}{|l|l|l|}
\hline Name & Equation & Remarks \\
\hline Reynold & $\eta_{0}=b \cdot e^{-a T_{a}}$ & Early equation; is accurate for very limited temperature range \\
\hline Slotte & $\eta_{0}=\frac{a}{\left(b+T_{A}\right)^{c}}$ & Reasonable; useful in numerical analysis \\
\hline Walther & $\left(v_{0}+a\right)=b \cdot d^{\frac{1}{T_{A}^{2}}}$ & Is the basis of the ASTM diagram of viscosity-temperature dependence \\
\hline Vogel & $\eta_{0}=a \cdot e^{\frac{b}{\left(T_{A}-c\right)}}$ & Accurate; very useful in engineering calculation \\
\hline
\end{tabular}

Where: a, b, c, $d$ are constants; $v_{0}$ is the kinematic viscosity at atmospheric pressure, expressed in $\left[\mathrm{m}^{2} / \mathrm{s}\right] ; \eta_{0}$ - is the dynamic viscosity at atmospheric pressure, expressed in [Pas]; $\mathrm{T}_{\mathrm{A}}$ - is the absolute temperature expressed in $[\mathrm{K}]$.

Oil viscosity increases as the pressure increases.
The best-known equation describing this dependence is the Barus equation [14]:

$$
\eta=\eta_{0} \cdot e^{\alpha \cdot p}
$$

Where: $\alpha$ is the viscosity-pressure coefficient that depends on temperature. Table 3 gives the values of this coefficient $\alpha$ for mineral oil HLP 32.

Table 3. Viscosity-pressure coefficient as a function of temperature

\begin{tabular}{|l|l|l|l|l|l|l|l|l|l|l|l|}
\hline $\mathrm{t}\left[{ }^{\circ} \mathrm{C}\right]$ & 0 & 10 & 20 & 30 & 40 & 50 & 60 & 70 & 80 & 90 & 100 \\
\hline$\alpha\left[10^{-2} \mathrm{~Pa}^{-1}\right]$ & 3,268 & 2,900 & 2,595 & 2,339 & 2,121 & 1,933 & 1,770 & 1,626 & 1,499 & 1,385 & 1,283 \\
\hline
\end{tabular}


Another equation that expresses the viscosity dependence on pressure is the following:

$$
\eta=\eta_{0} \cdot a^{\left(\frac{P}{P_{0}}-1\right)}
$$

Where $a=1,003$ for all mineral oils.

Equation (21) becomes now:

$$
\frac{\eta}{\eta_{\mathrm{O}}}=1+0.0003 \cdot \frac{\Delta P}{P_{\mathrm{O}}} \quad \text { or } \quad \frac{\eta}{\eta_{\mathrm{O}}}=0.003 \cdot \frac{\Delta P}{P_{\mathrm{O}}}
$$

For $\Delta \mathrm{P}=50$ bar we have $\Delta \eta / \eta_{0}=0,15$ so a very low viscosity variation.

\section{Conclusions}

In the case of hydraulic systems, a large number of fluids are used as working medium, ranging from water to molten metals, each with extremely different properties and characteristics.

In-depth knowledge of the basic properties of fluids is of great importance in the case of the theoretical analysis of such a system.

Theoretical analysis involves establishing equations that describe the dynamics of the system, even if it is not physically realized.

At the same time, it is well known that a hydraulic system is characterized by a large number of variables and interactions that are difficult to quantify, which is immune to a rational and systematic approach to modeling and simulation problems.

These systems are characterized by complex processes of fluid dynamics and thermo-technics, which is why the corresponding mathematical models are strongly nonlinear.

\section{References}

[1] M. Avram, Actionari hidraulice si pneumatice (Hydraulic and pneumatic systems), Bucharest, Ed. Universitara, 2005.

[2] D. Tataru, Tehnica simularii. Aplicatii in biologie. Aspecte teoretice (Simulation techniques, applications in Biology), Iasi, Ed. Stef, 2010.
To highlight this, the exponential term of the relationship (25) is processed as follows:

$$
a^{\left(\frac{P}{P_{0}}-1\right)}=(1+0.003)^{\frac{P-P_{0}}{P_{0}}}=1+0.003 \cdot \frac{\Delta P}{P_{0}}
$$

[3] D. Valer, Proiectarea sistemelor mecatronice (Mechatronic system design), Timisoara, Ed. Politehnica, 2007.

[4] J. A. Sarabando et al., Thermodyn. J Chem, 131, 2019 , p. 303.

[5] J. A. Österreicher, M. Kumar, A. Schiffl, S. Schwarz, and G. R. Bourret, Mater. Sci. Eng.A, 687, 2017, p. 175.

[6] J. Li, J. Zhou, A. Feng, S. Huang, X. Meng, and Y. Sun, Mater. Sci. Eng. A, 734, 2018, p.291.

[7] Q. Wang, L. Zeng, H. Ding, R. Chen, J. Guo, and H. Fu, Mater. Sci. Eng. A, 758, 2019, p.19.

[8] C. Balan, Lectii de mecanica fluidelor (Fluid mechanics lessons), Bucharest, Editura Tehnică, 2003.

[9] H. E. Merrit, Hydraulic control systems, New York, John Wiley \& Sons Inc, 1967.

[10] W. Backé, Ölhydraulik und Pneumatik, Grundlagen und Entwicklungstendenzen in der Ventiltechnik, Bremen, 1990.

[11] C. Nitu and M. Avram, Rev. Constructia de maşini (Automobile construction), 7, 1993, p. 54.

[12] P. Keith and B. Hodges, Hydraulic Fluids FILTERABILITY, vol. pure mech. London, 1996.

[13] G. W. Stachowiak and A. W. Batchelor, Engineering tribology, 4th Editio, Oxford, Butterworth-Heinemann, 2014.

[14] P. W. Gold, A. Schmidt, H. Dicke, J. Loos, and C. Assmann, J. Synth. Lubr.,18, 2000, p. 51.

[15] M. Jelali and A. Kroll, Hydraulic Servo-Systems. Modelling, Identification and Control, London, Springer, 2012.

[16] M. M. Budeanu, S. Radu, V. Dumitrescu, Rev. Chim. (Bucharest), 68, 2017, p. 1177.

[17] M. M. Budeanu, V. Dumitrescu, Rev. Chim. (Bucharest), 61, 2010, p. 322. 